\title{
MOTIVE DES AUSBRUCHS UND DES AUFBRUCHS IN DEN HÖRSPIELEN INGEBORG BACHMANNS
}

ABSTRACT. Leaving of the society in the radio plays of Ingeborg Bachmann

Bachman wrote only three radio plays, all of them in the fifties of the XXth century. In spite of different settings, all of them have the same topic: escape from society (either into dream or to an island or to the isolation of a couple wishing to live only for love) and utopia. In the first and third play the male protagonists fail however, unable to give up their bourgeois lives. In the third play the female protagonist is a victim of her ability to love without restrictions. This locates The Good God of Manhattan close to the feminist prose of Bachmanns later years. In the second radio play, The Cikadas, the existence on an island separated from society is judged in the negative. All attempts to win utopian happiness end with defeat; although they are valued differently, they show that in spite of all efforts mankind is not able to reach the ideal of truth and love.

KEY WORDS: Bachmann, radio plays, society, escape, leaving, freedom, love, dream, utopia

Ingeborg Bachmann hat nur drei Hörspiele, und zwar in der Zeitspanne von 1951-1957 ${ }^{1}$, geschrieben. Es ist anzunehmen, dass dies mit ihrer Arbeit im ScriptDepartment des der amerikanischen Besatzungsbehörde unterstellten Senders RotWeiß-Rot (Höller, 1999, S. 47) zusammenhing und generell im Kontext der Blütezeit dieser Gattung nach 1945 erfolgte. Albrecht und Göttsche berichten im Bachmann-Handbuch, dass dieser österreichische Sender im Zentrum der Interessen der amerikanischen Medienpolitik in Bezug auf den Kalten Krieg stand. Dort lernte auch Ingeborg Bachmann, Unterhaltung zwecks Umerziehung (der ehemaligen Nazis und Nazi-Mitläufer) und österreichischer Identitätsbildung einzusetzen. Die genannten Autoren gehen begründeterweise davon aus, dass Bachmann ,in ihren Hörspielen

\footnotetext{
${ }^{1}$ Ich führe sie nach Bachmann, Werke, Bd. 1: Anmerkungen, S. 661-662, an: Ein Geschäft mit Träumen, entstanden 1951, gesendet 1952, veröffentlicht zuerst 1976 (in Die Hörspiele); Die Zikaden, entstanden 1954, gesendet und veröffentlicht 1955; Der gute Gott von Manhattan, entstanden 1957, gesendet und veröffentlicht 1958.
} 
auf ähnliche Weise versucht, politische Fragen in einem politischen Kontext zu behandeln, ohne daß ihr Publikum das unbedingt bemerkt" (Albrecht \& Göttsche, 2013, S. 85).

Wie man aus dem Band von Heinz Schwitzke, dem Leiter der Hörspielabteilung beim Nordwestdeutschen Rundfunk in Hamburg, erfährt, wurde diese Gattung kurz nach der Eröffnung des ersten deutschen Rundfunksenders im Jahre 1923 eingeführt. Allerdings waren die ersten Programme der ", Sendebühne" einfach im Rundfunk gesendete Dramen, mit Schillers Wallensteins Lager oder Lyriklesungen (Hofmannsthal) angefangen. 1925 wurde das erste deutsche Original-Hörspiel gesendet: Spuk, nach Motiven von E. T. A. Hoffmann (Schwitzke, 1960, S. 9-10). Laut Schwitzke erlebte das Hörspiel schon an der Wende der 20er und 30er Jahre des 20. Jahrhunderts seinen ersten Höhepunkt. Damals konkurrierten zwei Auffassungen der Gattung: die von Hermann Pongs, dem Autor der ersten deutschen Monographie der Gattung, Das Hörspiel (1930), welcher durch das Hörspiel ein Gemeinschaftsgefühl erzeugen und stärken wollte (Pongs, 1930, S. 5, angeführt nach Keckeis, 1973, S. 8), was dann durch die Übernahme dieses Genres durch die Nazis zum ethischen Verfall des Hörspiels bis 1945 führen sollte, und die von Richard Kolb, dem Autor der Arbeit Das Horoskop des Hörspiels (1932), der meinte, dass das Hörspiel „mehr die Bewegung im Menschen, als die Menschen in Bewegung zu zeigen" (Keckeis, 1973, S. 9) berufen sei, wobei er aus der akustischen Unmittelbarkeit und psychologischen Wirkung des Hörspiels die Verinnerlichung dieser Gattung als natürlich postulierte. Diese Möglichkeit, das menschliche Innere zum Ausdruck zu bringen, eine „,innere Bühne" aufzustellen, gewann besonders viele lyrische Dichter für die neue Gattung. Wie Keckeis meint, „[hatten] die Ideen Pongs (...) zur Folge, dass das Hörspiel seinen Themen nach vielfach den Platz der Gegenwartsdramatik einnahm - bereits vor 1945 mit gleichgerichteten Vorzeichen, besonders aber in der Nachkriegszeit in Ermangelung von Theater und Film“" (Keckeis, 1973, S. 10).

Es ist allgemein bekannt, dass die Blütezeit des Hörspiels nach 1945 zum Teil aus dem Papiermangel für Druckerzeugnisse resultierte; Keckeis weist außerdem auf die zerstörten Theater und den Mangel an Filmen hin. Bartsch zufolge war vor dem massenhaften Erscheinen der Taschenbücher und dem Einsetzen der Vorherrschaft des TV das Radio das einzige Massenmedium (Bartsch, 1979, S. 316). Der Klassiker der Hörspielforschung Schwitzke betont die positiven Auswirkungen dieser Situation, nämlich die unerhörte Entwicklung des Mediums Rundfunk und seiner Möglichkeiten, die einen besonderen Anreiz und eine Herausforderung für die Dichter darstellten. In seiner Definition des Hörspiels hebt er die Einzigartigkeit dieser Gattung hervor:

Versteht man das Hörspiel - einerseits als Mischung von lautwerdenden und sogleich verlöschenden Worten und Klängen durch das Mittel technisch-elektrischer Produktion - andererseits als ganz unkörperliche, bloß spirituelle ,Anschauung' im Innern des Zuhörers, so kennt man eigentlich bereits alle Gründe seines Reichtums und sein ganzes, wunderbar einfaches und einheitliches Kunstprinzip. (Schwitzke, 1960, S. 18) 
Historiker des Hörspiels wie Keckeis für das deutsche Hörspiel oder Heger für das österreichische erklären, dass diese Tendenz zur „inneren Bühne“ der Figuren und der Empfänger etwa bis zum Anfang der 60er Jahre gedauert habe. 1961 habe ja Friedrich Knilli mit seinem Buch Das Hörspiel sich prinzipiell gegen die Verwendung akustischer Mittel „zum Aufbau einer fiktionalen Wirklichkeit“ und „für deren Verselbständigung aus dem Zwang jeglicher Abbild-Funktion“ (Keckeis, 1973, S. 12) ausgesprochen. In der zweiten Hälfte der 50er erfolgt dann eine Wende in der Entwicklung des westdeutschen und österreichischen Hörspiels, die aber sicher nicht isoliert, sondern im Zusammenhang mit der gesellschaftlichen Entwicklung, etwa mit der bald erfolgenden Studentenrevolte von 1968 oder mit der Entwicklung der Konkreten Poesie, zu betrachten ist. Es entstand das sog. Neue Hörspiel, und seine Theoretiker und Praktiker kamen dem herkömmlichen Hörspiel ins Gehege (Heger, 1977, S. 228-230). Hans Höller weist überzeugend nach, wie ungerecht die Vorwürfe der Verinnerlichung als Flucht vor sozialen Problemen, die damals das ,traditionelle" Hörspiel generell betrafen, im Falle von Bachmanns Hörspielen waren (Höller, 1987, S. 111-112). An der Debatte zweier Autoren, Wolf Wondratscheks, der anachronistisch Ingeborg Bachmann verurteilte, und Jürgen Beckers, der sie im Kontext ihrer Zeit sah, merkt man, wie viele Missverständnisse zwischen den Vertretern des verdienten ,poetischen Parabelspiels“ (Bezeichnung von Heger, 1973, S. 147 u. 164) aus der Zeit von 1945-1960 und denen des „Neuen Hörspiels“ bestanden ([Wondratschek] \& Becker, 1989, S. 113-118) ${ }^{2}$. Vielleicht hat Ingeborg Bachmann auch aus diesem Grund dann keine Hörspiele mehr geschrieben.

Wenn man ihre drei Hörspiele thematisch untersucht, sieht man, dass sie trotz Differenzen im geographischen und sozialen Raum der Texte darin das gleiche Motiv des Ausbruchs aus der zeitgenössischen Gesellschaft und des Aufbruchs zur Utopie abwandelt. Höller betont zu Recht die Beziehung zwischen diesen Hörspielen und den dazwischen entstandenen Gedichtbänden und weist auf einen Wandel im Umgang mit dem Utopiemotiv bei der Dichterin hin (Höller, 1989, S. 72-75). Meinem Anliegen soll allerdings das bekannte Motto der Autorin aus ihrer Rede zur Verleihung des Hörspielpreises der Kriegsblinden, Die Wahrheit ist dem Menschen zumutbar, voranstehen:

Es ist auch mir gewiß, daß wir in der Ordnung bleiben müssen, daß es den Austritt aus der Gesellschaft nicht gibt und wir uns aneinander prüfen müssen. Innerhalb der Grenzen aber haben wir den Blick gerichtet auf das Vollkommene, das Unmögliche, Unerreichbare, sei es in der Liebe, der Freiheit oder jeder reinen Größe. (Bachmann, 1978, Bd. 4, S. 276)

Wie sie weiter ausführt, komme es darauf an, das Unmögliche zu verfolgen, auch wenn man es nie erreiche, denn je näher man ihm komme, desto mehr entferne

\footnotetext{
${ }^{2}$ Es ist mir leider nicht gelungen, an das Original der Debatte in Merkur 24, Jg. 1970, S. 190-194, heranzukommen.
} 
sich der Horizont. Es komme auf dieses Spannungsverhältnis an, auf den Versuch, den Möglichkeitssinn anzuwenden (Bachmann, 1978, Bd. 4: der Essay Ins tausendjährige Reich, S. 24-28, und der Radioessay Der Mann ohne Eigenschaften, S. 80-102). Bartsch bemerkt zu Recht, dass sie sich vom Musilschen „Möglichkeitssinn“ und natürlich dem ,anderen Zustand“ sowie von Wittgensteins Philosophie des Sagund Unsagbaren inspirieren lässt (Bartsch, 1979, S. 315). In allen drei Hörspielen der österreichischen Dichterin treten Figuren auf, die diesen Aus- und Aufbruch, ob bewusst oder nur unbewusst im Traum, begehren und ausprobieren, auch wenn er zum Scheitern verurteilt ist. Alle drei Texte bedienen sich dabei einer Art Rahmenhandlung oder Parallelhandlungen. Auch die Musik und die Straßengeräusche spielen bei der Behandlung des Motivs des Aus- und Aufbruchs eine wichtige Rolle.

Ein Geschäft mit Träumen, das früheste Hörspiel Bachmanns, offensichtlich von Eichs Träumen inspiriert (Höller, 1987, S. 75-77), weist noch österreichisches Lokalkolorit auf. Die realistische Handlung stellt das Ende eines langen Tages in einem Büro eines Konzerns, den Abendspaziergang zweier Mitarbeiter und dann den nächsten Morgen wieder im Büro dar. Der Protagonist Laurenz ist ein Untergebener, der allen zur Hand geht, alles erledigt, Schreibmaschinen zur Reparatur bringt, sich um defekte Wasserhähne kümmert usw. Er kommt immer als Erster zur Arbeit und verlässt als Letzter das Büro. Er hat Schwierigkeiten, sich zu äußern, und selbst die Sekretärin Anna, die ihm gefällt und im Traum seine Partnerin ist, hat den Eindruck, mit ihm nicht mehr als zehn Sätze gewechselt zu haben (GmT, S. 183). Die Interpreten pflegen an diesem Beispiel seine subalterne Stellung und den unbarmherzigen kapitalistischen Arbeitsprozess zu betonen (z. B. Bartsch, 1979, S. 317). Auch Anna ist ihrerseits nicht sprachmächtig, zwar schwatzt sie viel, sie schöpft aber ihr Wissen aus Illustrierten und plappert nach, was sie dort gelesen hat. Eines Abends, als Laurenz ausnahmsweise ein bisschen früher weggeht, bittet ihn ein Kollege, der Beamte Mandl, ihm beim Einkauf eines Geburtstagsgeschenkes für seine Frau behilflich zu sein. Laurenz erweist sich als unfähig dazu, will aber auf Herrn Mandl am Donaukanal warten; dabei weckt ein unansehnlicher, schlecht beleuchteter Laden sein Interesse, wobei ihn eine immer intensivere Musik in dessen Richtung leitet. Es erweist sich, dass dieser Laden „ein Geschäft mit Träumen“ ist. Schon in diesem Hörspiel werden Effekte des Straßenlärms, der lauten, aufdringlichen Werbung und der Stimmen der Passanten, die Belangloses reden, verwendet, wie sie dann etwa im Gedicht Reklame aus der Sammlung Die Anrufung des Großen Bären (1956/1957) (Bachmann, 1978, 1, S. 114) oder im letzten Hörspiel der Autorin vorkommen. Sie bilden einen Kontrapunkt zur magischen Binnenhandlung und zur Musik, die Laurenz anzieht. Der Verkäufer erklärt ihm, dass in seinem Laden mit Träumen gehandelt wird, und lässt ihn drei davon in der Dunkelheit ausprobieren. Der Inhalt dieser Träume bedeutet einen unbewussten Ausbruch aus dem monotonen, bescheidenen Alltagsleben des Protagonisten. Man glaubt einer Anwendung von Freuds Lehre vom Unbewussten und seiner Traumdeutung zu begegnen. 
Im ersten Traum werden Laurenz, Anna und Mandl vom Generaldirektor verfolgt, der in einem Tunnel einen rasenden Zug gegen sie sausen lässt. Die Interpreten pflegen diesen Traum mit der Wirklichkeit der Nazizeit, ihrem Missbrauch der Technik und überhaupt den Gefahren der technischen Entwicklung, insbesondere angesichts des Kalten Krieges, in Verbindung zu bringen. Offensichtlich werden in diesem Traum die Ängste des kleinen Mannes artikuliert. Laurenz kann diesen Traum nicht aushalten, er bittet den Verkäufer um die Unterbrechung der Schau. Der zweite Traum, der ihm angeboten wird, zeigt den Ausbruch als eine Umkehrung der gesellschaftlichen Position der Agierenden. Laurenz wird $\mathrm{zu}$ einem SuperGeneraldirektor, der den Konzern ,zu einer der gigantischsten, einer noch nie dagewesenen weltumspannenden Organisation gemacht" (GmT, S. 202) hat. Anna ist seine Geliebte, und der ehemalige Generaldirektor muss ihm gehorchen. Da sieht man den vielleicht unbewussten Traum des kleinen Boten von der Umkehrung der sozialen Hierarchie und von der Rache an denjenigen, die ihn bisher gedemütigt haben. Aber man sieht in diesem Traum auch, dass er rasch jeder Unterhaltung und jeder Person überdrüssig wird. In dieser Laune erklärt er einen Krieg gegen die ganze Welt und rast zusammen mit Anna mit einer Rakete zum Mond. Bald aber langweilt ihn auch die Liebe dieser Frau, daher möchte er sie auf dem Mond zurücklassen. Als sie hört, dass sie ihm nichts bedeuten kann, springt sie aus der Rakete in den Weltraum. Laurenz ist zuerst mit seiner Position in diesem Traum zufrieden, dann aber wird er ihm unheimlich, und er lässt auch diese Präsentation unterbrechen. Erst im dritten Traum sehen wir eine positive Utopie, die zuerst von Anna und dann auch von Laurenz angestrebt wird. Laurenz liebt in diesem Traum Anna und ist verzweifelt, dass sie auf einem Schiff wegfahren will. Das ist das Motiv, das wir aus späteren Gedichten Bachmanns, etwa aus Ausfahrt, dem Eröffnungsgedicht in der ersten Gedichtsammlung Bachmanns, Die gestundete Zeit (1953) (Bachmann, 1978, 1, S. 28) kennen. Im Hörspiel widersetzen sich Annas Hoffnungen auf eine andere Welt den kleinbürgerlichen Lebenswünschen des Laurenz. Laurenz stellt sich sein Glück mit Anna auf folgende Weise vor:

\footnotetext{
Anna, ich werde nicht in die Berge fahren. ${ }^{3}$ Wir werden um unser Geld ein kleines Haus kaufen. Verlaß das Schiff, Anna, spring, lauf! Spring ab, komm zurück! Wir werden Blumen in unserem Garten haben, und ich werde dir einen blauen Luftballon kaufen, wir werden Wein trinken, und ich werde dich mit weißen Wolken zudecken! Lauf und spring ab! ( GmT, S. 208)
}

Anna lässt sich aber nicht von diesem bürgerlichen Glück verlocken, sie liebt die Weite des Meeres und das Wagnis bis zum Tode. Das Meer symbolisiert hier wie oft bei Bachmann die Unendlichkeit, die Aussicht auf ein utopisches Glück, das allerdings mit dem Leben bezahlt werden müsste. Denn Anna entgegnet auf die wiederholten Aufforderungen und Warnungen ihres Partners:

\footnotetext{
${ }^{3}$ Was bisher seine einzige Erholung war, für die er das ganze Jahr über Geld sparte (GmT, S. 188).
} 
Ich liebe die wundervollen Lieder der Matrosen, ich liebe das Meer und die Ferne, die Unendlichkeit und die Gefahr. Und ich hasse die Stadt mit den Dächern, die auf meine Schultern drücken, und die Umarmungen der kleinen grauen Tränen am Ufer [ein pars pro toto für Laurenz' Traurigkeit über ihren Verlust. - M.K.], ich hasse das Leben und die Menschen, die in die Berge fahren wollen, sich ein Haus bauen und mir im Garten des Abends die Augen mit Küssen bedecken ... Aber ich liebe den Tod. (GmT, S. 209)

Kurt Bartsch macht in diesem Zusammenhang zu Recht darauf aufmerksam, dass hier die höchste Erfüllung wie in Wagners Tristan und Isolde zugleich den Tod bedeutet (Bartsch, 1997, S. 80). Und tatsächlich geht das Schiff mit dem ironischen Namen „Securitas“ unter. Während der Verkäufer in diesem Moment den Traum abstellen möchte, protestiert Laurenz, dass es sein Traum sei, und bekennt sich damit zu der am Tage nicht einmal vor sich selbst eingestandenen Liebe zu Anna. Er ist ziemlich ratlos, wie er sich in dieser Situation verhalten soll, um Anna zu retten. Als die Traumprojektion wieder eingeschaltet wird, ist Anna schon tot und ruht ertrunken neben den Körpern der toten Matrosen. Laurenz überwindet nun seine Begrenzungen, kommt zu ihr und kann auf einmal auch die bei Bachmann so wichtige neue Sprache, die Sprache der Liebe und der Unendlichkeit sprechen. Er findet die Geliebte auf dem Meeresgrund und will sich für immer mit ihr vereinigen:

Wir werden für immer beisammen sein, und nichts soll uns trennen. Unser Haus wird auf den Quellen des Lebens stehen, wir werden in allen Geheimnissen seiner wechselnden Mauern wohnen. Und in den Spiegeln des Grundes kann ich deine schöne Gestalt vertausendfältigt sehen. (GmT, S. 212)

Diese Stimmung wird durch den Sprechgesang der Sirenen und die verklingende Musik, die einen Welleneffekt nachahmt, gesteigert. Die Liebenden bekennen einander ihre Liebe, und Laurenz erwacht. Er möchte diesen Traum erwerben, es stellt sich aber heraus, dass der kapitalistischen Wirklichkeit entgegen sich nicht alles mit Geld bezahlen lässt. Träume kosten Zeit, und zwar würde dieser schöne Traum einen Monat seines Lebens kosten. Da erweist es sich, dass die Traumvision Laurenz nicht verändert hat. Er meint kleinlich, dass er keine Zeit zur Verfügung habe, er müsse arbeiten, nicht einmal seinen Urlaub ist er zu opfern bereit. Er reagiert erschrocken, als sich zeigt, dass es schon Morgen ist und er zu spät zur Arbeit kommt. Und auch dort hat sich nichts verändert, er wird von Anna nörgelnd begrüßt. Nur als Herr Mandl erzählt, dass er für seine Frau ein Seidentuch erworben habe, sagt sie mit verträumter Stimme: „Die Seidentücher beim Ferez, die sind ja entzückend. (...) Beim Ferez, die sind ja traumhaft" (GmT, S. 216). Damit schließt sich der Rahmen mit einer Aussage aus der Sprache der Werbung und des Konsums, die klarstellt, dass Anna ebenso wie Laurenz in kleinbürgerlichen Vorstellungen befangen bleibt, dass beide nicht zueinander kommen und dass Laurenz' Traum lediglich ein Traum, eine Illusion war.

Während im ersten Hörspiel der Ausbruch aus der konventionellen Klassengesellschaft, wenn auch nur im Traum, glückt und als eine positive Lösungsmöglich- 
keit gezeigt wird, der der Protagonist nicht standzuhalten vermag, führt die Auswanderung der „Schiffbrüchigen“ auf die „Insel“ im Hörspiel Die Zikaden zu keinen positiven Resultaten. Zwischen den beiden Hörspielen liegt nämlich, worauf Hans Höller hingewiesen hat, der erste Italienaufenthalt Ingeborg Bachmanns in den Jahren 1953-1957, von denen sie den Anfang auf der Insel Ischia in der Nähe des Komponisten Hans Werner Henze verbrachte (Höller, 1999, S. 83-87). Der mediterrane Schauplatz des Hörspiels steht offensichtlich in biographischem Zusammenhang mit dieser Flucht nach dem Süden. Höller (1987, S. 100) führt ein Zitat aus Henzes Statement Die Krise des bürgerlichen Künstlers an, wo dieser erklärt, dass er wie viele andere nach dem Zweiten Weltkrieg aus Deutschland weggegangen sei, weil dort überall das Erbe des Faschismus spukte. Erst mit der Zeit habe er in Italien bemerkt, dass er sich auf seiner Insel (ob wörtlich oder metaphorisch verstanden) immer mehr von der Gesellschaft isolierte und seine Musik ins Private driftete. Ähnlich wird es seiner Freundin Ingeborg Bachmann gegangen sein. Andererseits legt gerade der Topos der Mittelmeerinsel Anspielungen auf die antike Kultur nahe. Besonders drückt sie sich in dem Titel des Hörspiels Die Zikaden und dem Leitmotiv ihres „wilden, frenetischen Gesang[s]“ (DZ, S. 221) aus, das am Ende des Textes durch den Erzähler in Anknüpfung an Platos Gastmahl folgendermaßen erklärt wird:

Denn die Zikaden waren einmal Menschen. Sie hörten auf zu essen, zu trinken und zu lieben, um immerfort singen zu können. Auf der Flucht in den Gesang wurden sie dürrer und kleiner, und nun singen sie, an ihre Sehnsucht verloren - verzaubert, aber auch verdammt, weil ihre Stimmen unmenschlich geworden sind. (DZ, S. 268)

Erika Tunner weist darüber hinaus auf die Konnotationen mit der Insel der Seligen hin ${ }^{4}$ und mit der Insel als Ort der Utopie wie in Defoes Robinson Crusoe, auf dessen Namen Bachmann bewusst mit der Bezeichnung einer der Figuren anspielt (Tunner, 1986, S. 82-84). In diesem Hörspiel begleitet die ganze Zeit über der wortlose Zikadengesang die Handlung und bildet eine Parallele zu ihr, wenn man eben auf den antiken Mythos rekurriert. Roland Heger hält in seiner Monographie Das österreichische Hörspiel dieses Werk Bachmanns für „das formal vollendetste“ [!] von ihren „poetischen Parabelspielen“ (Heger, 1977, S. 164 u. 174). Als einziges der Hörspiele Bachmanns bedient es sich einer Erzählerfigur, die viel Distanz zu den übrigen Gestalten schafft und gleichzeitig durch ihre Einleitung, ihre Kommentare und ihre Pointe eine Art Rahmenhandlung bildet. Durch den Erzähler erfahren wir zunächst, dass alle diese Flüchtlinge, die auf der Insel ein Asyl suchen, „Schiffbrüchige" sind. Diesem Motiv hat Erika Tunner ihren bereits genannten Aufsatz gewidmet. Sie sind schiffbrüchig nicht im wörtlichen Sinne, denn sie haben Geld,

\footnotetext{
${ }^{4}$ Obwohl Bachmann hier in der Bezeichnung der Gefängnisinsel, aus der zu entrinnen es keine Hoffnung gibt, als „Ort der Erlösung“ eher auf die tückische Ironie des „Arbeit macht frei“ im KZ Auschwitz anspielt.
} 
Pässe und all ihre Habe, die sie mitnehmen wollten, aber sie konnten sich im Leben in ihrer Heimat nicht zurechtfinden und sind daher auf die Insel geflohen.

Der Erzähler sagt: „Und die meisten auf dem Schiff sind Fremde, mit Gesichtern, in die viele Grenzübertritte gestempelt sind. Sie kommen aus der ganzen Welt. Und ich kenne sie alle. Denn es sind immer die Schiffbrüchigen, die auf Inseln Zuflucht suchen.“ (DZ, S. 222) Nur über einen dieser Schiffbrüchigen, über den Journalisten Benedikt, erfährt man, dass er während der faschistischen Zeit auf die Insel kam, weil er vom Militär desertierte. Andere scheinen aus persönlichen, unpolitischen Gründen die Insel gewählt zu haben, etwa Mrs. Brown, die eine Abtreibung und den Verlust ihrer schönen Stimme nicht verschmerzen kann, ihr Mann, der immer wieder nach dem versunkenen Sohn sucht, Jeanette, die sich mit dem Altern nicht abfinden kann oder Salvatore, der durch seinen Alkoholismus als Maler versagt hat und sich nun Inspiration von den Landschaften der Insel erhofft. Auch der letzte der Flüchtlinge, der jugendliche Stefano, ist ausgerissen, weil er Angst vor der Reaktion der Eltern auf sein Zeugnis hatte und ein großes Abenteuer unter den ,Wilden' erleben wollte. Alle diese Gestalten, derer neun vorgeführt werden, ähneln einander in ihrem Wunsch, das im realen Leben Unerreichbare auf der Insel nachzuholen. Auch der etwas näher gezeigte Mann, der anstatt mit seinem Namen ironisch „Robinson“ ${ }^{\text {"5 }}$ genannt wird, ist ein zentrifugaler Wanderer, der die Heimat, seine Frau, die ihm Briefe schreibt, um ihn zur Heimkehr aufzufordern, und seine alltäglichen Pflichten flieht. Er behauptet zwar, dass die Gesellschaft sich „fortgesetzt an [s]einem Leben vergriffen hat" (DZ, S. 259), aber es gibt keine Beweise, dass es tatsächlich so ist.

Die einzige Ausnahme ist, ebenfalls ironisch genug, der Sträfling (,der Gefangene“) von der „Insel der Erlösung“, dem es gelungen ist, nackt schwimmend die Distanz zwischen den beiden Inseln zu überbrücken, und der bei Robinson Hilfe sucht. Unwillkürlich denkt man dabei an den schiffbrüchigen Odysseus, der nackt den Strand der Phaiaken erreicht und von der Prinzessin Nausikaa Hilfe erhält. Charakteristisch für alle diese Figuren ist die Nichtrealisierbarkeit ihrer Wünsche, selbst auf der holden Insel im Mittelmeer, und ihre gesellschaftliche Isolierung, in der jeder nur für sich selbst Erfüllung sucht, auch wenn manche von ihnen mit anderen Ankömmlingen gesellig verkehren, wie Prinz Ali, der Empfänge gibt, oder Jeanette, die sich als Kosmetikerin betätigt. Kontrapunktisch zu ihnen steht die Gestalt des schönen jungen Einheimischen Antonio, der seinen Lebensunterhalt damit verdient, den Fremden auf jede mögliche Weise behilflich zu sein, und der zuerst auf die Erwähnung ihrer Wünsche immer nur mit „Ja!“ antwortet, wenn sie sich aber in ihren Illusionen steigern, endlich ihr Quasi-Selbstgespräch mit einem energischen „Nein!“ unterbricht.

\footnotetext{
${ }^{5}$ Der Robinson des Prätextes von Defoe erlitt ja einen unfreiwilligen Schiffbruch und sehnte sich nach der Rückkehr.
} 
Auf der Insel scheint keine Zeit zu vergehen, jeder Tag ist wie der andere, es ist ein langweiliges, untätiges Paradies, ein Schlaraffenland, in das sie hineingeraten sind, das keinen befriedigt. Es gibt auf der Insel keine Liebe, nur Sex. Durch die Inselzeitung Benedikts erfahren sie lediglich lokale Neuigkeiten, die so zurechtgestutzt sind, dass sie niemandem wehtun. ${ }^{6}$ Nur der Gefangene, der ja durch den Zwang des Gerichts in jene Welt geriet, weiß den Wert der Gesellschaft, mit der er immer im Streite war, und des tätigen Lebens zu schätzen. Nicht er ist aus der Gesellschaft ausgebrochen, sie hat ihn ,versetzt, transferiert auf den Außenposten“ (DZ, S. 262). Er will die verlorenen Jahre, die er im Inselgefängnis verbracht hat, nachholen. Aber er weiß, dass er den geliehenen Anzug Robinsons nicht anziehen kann, weil er ihm nicht „passt“, er muss sein Leben leben und sich den zwei jungen Gendarmen stellen, die ihm gegen Ende des Hörspiels auf den Fersen sind.

Da hat Hans Höller offensichtlich recht, wenn er behauptet, dass sich hier Bachmanns Utopieauffassung geändert hat:

In einem Moment, wo es sich erweist, daß die Ausfahrt zu anderen Ufern eine Illusion ist, bricht das Hörspiel mit den alten Modellen der Raum-Utopien, da ,Schiff, ,Ausfahrt', ,Insel' das Ich nur außerhalb der Gesellschaft mit seinen alten Verletzungen allein lassen. Das Hörspiel (...) schließt mit einer Demontage eines nicht mehr haltbaren Utopie-Entwurfs an eines der letzten Gedichte des ersten Lyrikbandes an. (Höller, 1987, S. 104).

In der den Rahmen schließenden Aussage des Erzählers kann man die Dichterin, als deren Sprachrohr er fungiert, heraushören: „Die Insel und die Personen, von denen ich erzählte, gibt es nicht. Aber es gibt andere Inseln und viele Menschen, die versuchen, auf Inseln zu leben. Ich selbst war einer von ihnen (...)“ (DZ, S. 267). Das betont den parabelhaften Charakter des Hörspiels. Die Pointe bildet die Zikaden-Geschichte. Die Zikaden, die einst Menschen gewesen seien, waren so in ihren Gesang verliebt, dass sie aufhörten, Menschen zu sein. Auf diese Weise wird der Utopie der Einsamkeit des Künstlers und der Suche nach einer heilen insularen Welt abgesagt. Kurz vor dem Ende fordert der Erzähler die Liebhaber eines Inselparadieses zur Aktivität auf:

Nun? Hier ist eine Insel, und was willst du? Soll die Sonne das Messer ziehen und der Vulkan die Asche auf dein Haupt tun? Willst du nicht aufstehen und sehen, ob diese Hände zu gebrauchen sind? Oder willst du dir die Welt erlassen und die stolze Gefangenschaft? Such nicht zu vergessen! Erinnre dich! Und der dürre Gesang deiner Sehnsucht wird Fleisch! (DZ, S. 267)

So endet der Ausbruch aus der Gesellschaft mit einer Aufforderung zum Weg zurück. Der Erzähler habe ihn selbst durchgemacht, und Robinson tut ihn ebenfalls,

\footnotetext{
${ }^{6}$ Z.B.: Nachdem seine Frau Robinson eingeholt hat und er unfreiwillig die Insel verlässt, schlägt Benedikt Antonio als kleinen Witz vor: „, [... wir] nennen unseren Mann Robinson. Robinson hat dem Schiff gewunken. Robinson kehrt heim“ (DZ, S. 265).
} 
obwohl nicht freiwillig. Es gibt keine Möglichkeit, außer durch den Tod, der Gesellschaft und dem Dienst an ihr zu entsagen.

Das Scheitern eines Versuchs, allerdings in der Zweisamkeit der Liebe, wird ebenfalls im letzten Hörspiel der Autorin thematisiert, Der gute Gott von Manhattan. Dieses Werk gewann den ehrenvollen Hörspielpreis der Kriegsblinden und wurde wegen seiner formalen Vollendung am höchsten geschätzt, erfuhr aber während der Revolte des „Neuen Hörspiels“ auch scharfe Kritik (Höller, 1987, S. 72 u. 111-112; Bartsch, 1991, S. 75 u. 81; Koschel, \& v. Weidenbaum 1989, S. 115-116). Diesmal spielt die Handlung in Amerika auf der New Yorker Insel Manhattan, was wohl biographisch gesehen ein Ergebnis der USA-Reise anlässlich eines Seminars an der Harvard-Universität im Jahre 1955 war (Albrecht \& Göttsche, 2013, S. 92). Auch diesmal entwirft die Dichterin zwei Handlungsebenen, und zwar einen Rahmen mit der Schilderung eines Mordprozesses gegen den „guten Gott von Manhattan" sowie eine Reihe von Rückblenden, die zeigen, wie es zu diesem Mord gekommen ist. Diese Szenen sollen dem ,guten Gott“, der ein rigides Gesellschaftsprinzip repräsentiert und jeden Ausbruch aus der gesellschaftlichen „Ordnung“ mit dem Tode zu bestrafen sucht, als Rechtfertigung dienen. Schließlich erkennt der Richter, der das Urteil fällen soll, dass sie beide die gleiche Meinung teilen, nämlich dass eine Liebe, die Ausschließlichkeit für sich verlangt und sich nichts aus bürgerlichen Konventionen und sozialen Verpflichtungen macht, beseitigt werden muss, weil sie eine Gefahr für die bestehende Gesellschaft bedeutet. Der Richter spricht zwar kein entlastendes Urteil aus, er lässt aber den Angeklagten durch eine Hintertür entweichen, was das Stück mit einem offenen Ausgang enden lässt. Offen ist dieser Ausgang allerdings nur für den Antagonisten des Liebespaares, denn Jan wird dank seines Verrats gerettet und zu einem Leben „bei schlechter Laune und mit mäßigen Aussichten" (DgGvM, S. 327) verurteilt, und die liebende, ganz ihrer Leidenschaft hingegebene Jennifer wird zum Opfer eines Bombenattentats des „guten Gottes“.

Die Einwände Wolf Wondratscheks gegen dieses Hörspiel, dass Ingeborg Bachmann kein authentisches New Yorker Leben mit seinen sozialen Spannungen und politischen Protesten zeige ([Wondratschek]/Becker, 1989, S. 116), beruhen offensichtlich auf einem Missverständnis, denn gerade dort, wo in Tristan und Isolde ein kristallenes Bett in einer Waldgrotte als Symbol der alles ausschließenden Liebe erscheint, fungiert im Werk aus der Nachkriegszeit nach 1945 ein Hochhaushotel, mit den immer mehr der Liebe zuträglichen Zimmern auf immer höheren Stockwerken, als das gleiche, ins Moderne umgesetzte Symbol. Übrigens treten im Hörspiel die Episodenfiguren eines Bettlers, der über seinesgleichen in der Metropole New York spricht, einer wahrsagenden Zigeunerin und der Besitzerin eines elenden Stundenhotels auf, folglich werden die Protagonisten, bevor ihre Liebe den Zenit erreicht, wenn auch peripher, doch immerhin mit den Nachtseiten der Großstadt konfrontiert. Christine Lubkoll erkennt in ihrer Interpretation dieses Hörspiels richtig: „Im Hörspiel erscheinen die Macht des guten Gottes und die Utopie der 
Liebe gerade nicht scharf voneinander getrennt, es wird vielmehr gezeigt, wie das Liebeskonzept seinerseits von gesellschaftlichen Prinzipien durchzogen und von diskursiven Vorgaben bestimmt ist" (Lubkoll, 2002, S. 123).

Die Binnenhandlung lässt sich schnell nacherzählen. Der junge Europäer Jan, der von New York aus gerade ein Schiff in die Heimat nehmen will, begegnet auf dem Bahnhof der amerikanischen, schönen, mädchenhaft aussehenden Studentin Jennifer, die ihn schon im Zug bemerkt hat. Sie spricht ihn an, sie besichtigen zusammen New York und landen in einem schmutzigen Stundenhotel. Zunächst sieht es Jan nur als eine zu nichts verpflichtende Liebesepisode in einer fremden Stadt, aber beide verlieben sich immer stärker ineinander, immer leidenschaftlicher. Sie nehmen ein Zimmer im Hotel Atlantic City, und nachdem sie sich dort schon voneinander verabschiedet haben, erkennt Jan, dass er ohne Jennifer nicht leben kann. Sie bekommen ein höher platziertes Zimmer, zum Schluss gar ganz oben im 57. Stockwerk, was offensichtlich die Steigerung ihres Gefühls symbolisiert, und versprechen einander ewige Liebe. Ihre Leidenschaft wird so stark, dass der gute Gott sie zur Freude seiner Eichhörnchen zum Tode verurteilt und eine Bombe aus seinem Arsenal gegen sie vorbereitet. Allerdings trennen sich die Liebenden kurzzeitig, Jan soll seine Schiffskarte zurückgeben und dann immer bei Jennifer bleiben. Sie denken nicht daran, dass sie nur noch sehr wenig Geld haben, denken nicht an die Zukunft, für sie existiert nur die Gegenwart. Wie es Jan ausdrückt, beginnt die „Gegenzeit“ (DgGvM, S. 317). Aber wie es auch in späteren Werken Bachmanns zum Ausdruck kommt, erweist sich der männliche Protagonist als zu solch einer Liebe auf Dauer unfähig. Er betritt wie zufällig eine Bar, die ihn anlockte, angeblich nur, um einen Drink zu nehmen. Dort besinnt er sich wieder auf die Realität, auf die Zeit, die zuvor außer Kraft gesetzt worden war. Er fragt den Barmann nach der Uhrzeit, hört das laut eingestellte Radio, bittet um eine Tageszeitung und erfährt daraus, dass in seinem Land in Europa die Regierung gewechselt hat. Das genügt, damit er sich auf seine Vergangenheit und Zukunft besinnt, die Erde hat ihn wieder, wie es mit einem abgewandelten Faust-Zitat „der gute Gott“ ironisch kommentiert (DgGvM, S. 327). Er verweist wiederholt darauf, dass Jan schließlich nach Europa abreiste, ohne Jennifer wenigstens zu begraben. Für Jan ist das Vorgefallene wieder lediglich eine Liebesepisode, ein Abenteuer. Der Richter meint zwar ein wenig sentimental, dass Jan die Frau wohl nie vergessen werde (ebd.), aber wir können uns vorstellen, dass sie im besten Falle in seiner Erinnerung eine ähnliche Rolle spielen wird wie Musils Tonka in der gleichnamigen Erzählung: eine kleine warme Wolke sein, die ihn besser macht.

Es ist deutlich, dass Ingeborg Bachmann, die sich ja mit Musils Geschichte des „tausendjährigen Reiches“ der Liebe von Ulrich und Agathe im Mann ohne Eigenschaften für die Zwecke des Rundfunks beschäftigt hat, intertextuell auf das Motiv der „taghellen Mystik“, die das Liebeserlebnis der Geschwister kreiert, anspielt und 
ebenfalls zu zeigen versucht, dass solch eine ideale Liebe zum Scheitern verurteilt ist. Sie sagte ja selbst in der bereits zitierten Passage zur Verleihung des Hörspielpreises, dass „wir in der Ordnung bleiben müssen“. Als der Richter dem „guten Gott“ weiszumachen versucht, dass er viele gute Partnerschaften und Ehen kenne, die sich ihre Liebe bewahrt hätten, entgegnet dieser:

Ich gestehe Ihnen unzählige zu. Aber wer wird sich mit Menschen beschäftigen, die nach einem anfänglichen Seitensprung ohnehin Instinkt bewiesen haben. Die das bißchen anfängliche Glut zähmten, in die Hand nahmen und ein Heilmittelunternehmen gegen die Einsamkeit daraus machten, eine Kameradschaft und wirtschaftliche Interessengemeinschaft. ( DgGvM, S. 319)

Solch eine Liebe sei der Ordnung nicht gefährlich. Aber eine Leidenschaft, die nicht aufhört, die die Liebe zu den Sternen erhebt, schaffe ein Chaos wie vor dem ersten Schöpfungstag. „Ich glaube, dass die Liebe unschuldig ist und zum Untergang führt (...)“ (DgGvM, S. 318), mit diesem Oxymoron erklärt er sein Verhalten den absolut Liebenden gegenüber. Seine Handlanger, die Eichhörnchen, führen ein „Stück im Stück“ vor, wo sie die wichtigsten Liebespaare der Weltliteratur und ihren Untergang vorführen, was intertextuell auf das zwangsläufig tragische Schicksal der Liebenden hinweist.

Ingeborg Bachmann lässt die Frage nach dem Recht der kollidierenden Weltordnung bzw. Gesellschaftsordnung und der Liebe offen. Der Hörer oder Leser soll selbst seine Schlussfolgerungen ziehen. Nach dem Paradigma ihrer Prosa, von Undine geht über Malina bis zum Fall Franza, liebt der Mann weniger, ist der Mann nicht imstande, immer treu zu bleiben, immer der Liebe zu leben, was vielleicht notwendig ist, damit die Gesellschaft nicht aus dem Gleichgewicht kommt. Freilich ist der Rezipient oder zumindest die Rezipientin geneigt, sich mit Jennifers Sicht zu identifizieren. Sehr überzeugend finde ich allerdings die Einwände im Handbuch von Albrecht und Göttsche, wonach Jennifers unbedingte Liebe viele Fragezeichen hinterlässt. Sie will ja in der Liebe nicht sich selbst verwirklichen, sondern als Ergänzung zu Jan auftreten. Ihre Liebe hat sogar manche pathologischen Züge: Jennifer lässt ihren Partner mit den Nägeln tiefe Spuren in ihre Hände eingraben, wodurch sie ihn zu einem etwas sadistischen Verhalten animiert (DgGvM, S. 280 u. 283); er sagt, er möchte sie schlagen, weil sie auf ihn gehört habe und zuerst von ihm weggegangen sei (ebd., S. 301); sie sagt in der höchsten Hingabe, dass sie vor ihm auf Knien liegen und stets drei Schritte hinter ihm gehen werde (ebd., S. 321). Als die Gegenzeit beginnt, sagt sie zu ihm in der Liebesekstase: dass sie „(...) nach Erniedrigung (...) vergehe“, dass sie sich von ihm „hinrichten ließe (...) oder wegwerfen wie ein Zeug nach jedem Spiel, das [er ersinne].“ (ebd., S. 302) Das ist also keine Partnerschaft, keine Gleichheit in der Liebe, sondern ein eher masochistisches Gefühl und eine traditionelle Auffassung von der Verteilung der Geschlechterrollen. Jan reagiert darauf keineswegs mit Entrüstung, sondern fühlt sich 
stolz darauf, dass sie ihren Stolz für ihn aufgegeben hat (ebd., S. 302). Sie sprechen zwar nach der Auffassung Bachmanns eine „neue Sprache“, die Sprache der Liebe, aber diese Sprache operiert mit alten Bildern. Daher muss der These von Albrecht und Göttsche beigepflichtet werden:

Diese Beispiele legen nahe, daß Jennifer keine vor- und außersoziale Sexualität verkörpert, sondern vielmehr als Produkt eines schon existierenden Diskurses von ,gender ‘ und Sexualität zu verstehen ist. Innerhalb dieses Diskurses ist die weibliche Sexualität nur vorstellbar als eine, die einer des Mannes von vornherein unterlegen ist. (Albrecht \& Göttsche, 2013, S. 95)

Ich würde dies nicht nur auf die Sexualität, sondern auf die ganze Komplexität der dargestellten Beziehung zwischen Mann und Frau anwenden. Da Bachmannsche Frauenfiguren so denken, muss die Protagonistin des Malina-Romans in der Wand verschwinden, anstatt z. B. die erträumte Idylle mit Ivan zu erleben, und Franza muss ihrem Mann, dem „Fossil“, unterliegen. Ich bin mir nur nicht sicher, ob die Schlussfolgerung der Autoren des Bachmann-Handbuches nicht zu weit geht, der zufolge Bachmann kein „Entweder-oder“ wünscht. Die Polarität ist da. In allen drei Hörspielen gibt es den Gegensatz zwischen der Realität, der des Kalten Krieges, wie das oben genannte Handbuch zu Recht feststellt, und dem utopischen ,anderen Zustand“. Die Flucht aus der Realität zur angeblich absoluten Freiheit des Inseldaseins wird im Hörspiel Die Zikaden kritisiert, da sie dort keine Liebe, sondern den Egoismus der Einsamkeit, der monadenartigen Existenz - Freiheit nicht als Freiheit zur Liebe, sondern als Freiheit von sozialen Verpflichtungen - bedeutet. Im Geschäft mit Träumen und im Guten Gott von Manhattan ist es anders, sowohl die magische Wirklichkeit des „dritten Traums“ als auch die Liebesutopie Jans und Jennifers werden der gesellschaftlichen Realität gegenübergestellt als Ausdruck menschlicher Sehnsucht nach dem Ideal und nach einem nicht konformistischen Leben. Im ersten Hörspiel erweist sich dieses als unmöglich, da der Träumende dazu nicht fähig ist. Im Guten Gott von Manhattan bezahlt Jennifer dafür den höchsten Preis. Aber vielleicht ist es richtig, dass insgesamt die beiden Sphären als komplementär erscheinen, als das, was erst das Leben zu einem vollen macht. Allerdings ist die Autorin nicht geneigt, sich mit einem Kompromiss abzufinden. Das macht das Scheitern notwendig, aber es liegt nicht an der Liebe, sondern an der Gesellschaft, die keine Selbsterfüllung der Individuen ermöglicht.

\section{Bibliographie}

\section{Primärliteratur}

Bachmann, I. (1978). Ein Geschäft mit Träumen; Die Zikaden; Der gute Gott von Manhattan. In dies. Werke (Bd. 1, S. 179-327), München: Piper (Siglen GmT, DZ und DgGvM). 


\section{Sekundärliteratur}

Albrecht, M. \& Göttsche, D. (Hrsg.). (2013). Bachmann-Handbuch. Leben - Werk - Wirkung (Kap. Hörspiele, S. 83-96). Stuttgart: Metzler.

Bachmann, I. (1978). Werke. Bd. 4: Essays / Reden / Vermischte Schriften / Anhang / Photographie. München: Piper.

Bachmann, I. (1978). Werke. Bd. 1: Gedichte / Hörspiele / Libretti / Übersetzungen. München: Piper.

Barbe, J.-P. \& Wögerbauer, W. (Hrsg.). (1986). Ingeborg Bachmann. L'oeuvre et ses situations. Nantes: Université de Nantes.

Bartsch, K. (1979). Die Hörspiele Ingeborg Bachmanns. In K. Bartsch, D. Goltschnigg, G. Melzer \& W. Schober (Hrsg.), Die andere Welt. Aspekte der österreichischen Literatur des 19. und 20. Jahrhunderts (S. 311-334). Bern: Francke.

Bartsch, K. (1997). Ingeborg Bachmann (2. überarb. u. erw. Auflage). Stuttgart: Metzler. [Wondratschek, W.] \& Becker, J. (1989). War das Hörspiel der Fünfziger Jahre reaktionär?

Eine Kontroverse am Beispiel von Ingeborg Bachmanns Der gute Gott von Manhattan [1970]. In Ch. Koschel \& I. v. Weidenbaum (Hrsg.), Kein objektives Urteil - nur ein lebendiges? Texte zum Werk von Ingeborg Bachmann (S. 113-118). München: Piper.

Heger, R. (1977). Das österreichische Hörspiel. Wien: Wilhelm Braunmüller.

Höller, H. (1987). Ingeborg Bachmann. Das Werk. Von den frühesten Gedichten bis zum ,Todesarten Zyklus. Frankfurt a. M.: Athenäum.

Höller, H. (1999). Ingeborg Bachmann, Reinbek b. Hamburg: Rowohlt Taschenbuch.

Keckeis, H. (1973). Das deutsche Hörspiel 1923-1973. Frankfurt a. M.: Athenäum.

Koschel, Ch. \& v. Weidenbaum, I. (Hrsg.). (1989). Kein objektives Urteil - nur ein lebendiges? Texte zum Werk von Ingeborg Bachmann. München: Piper.

Lubkoll, Ch. (2002). Der gute Gott von Manhattan. In M. Mayer (Hrsg.), Interpretationen. Werke von Ingeborg Bachmann (S. 122-139). Stuttgart: Reclam.

Tunner, E. (1986). Von der Unvermeidbarkeit des Schiffbruchs. Zu den Hörspielen von Ingeborg Bachmann. In J.-P. Barbe \& W. Wögerbauer (Hrsg.), Ingeborg Bachmann. L'œeuvre et ses situations (S. 82-99). Nantes: Université de Nantes. 